\title{
ERRATUM
}

Patrick D. Weidman

\section{Erratum to: Offset rotating plates in a uniformly rotating fluid}

Published online: 28 August 2015

(C) Springer-Verlag Wien 2015

\section{Erratum to: Acta Mech 226, 1123-1131 (2015) \\ DOI 10.1007/s00707-014-1239-5}

We correct an error in the original paper concerning the angle of the locus of centers of rotation between two plates co-rotating at angular velocity $\Omega$ in a system uniformly rotating at angular velocity $\omega$. Without background rotation, Berker [1] noted that the curve describing the centers of rotation projected on the horizontal mid-plane forms $45^{\circ}$ logarithmic spirals and Weidman [2] erroneously stated that the same holds true with background rotation.

In [2], the problem is governed by the Reynolds number $R=h^{2} \Omega / v$ and the Coriolis parameter $\sigma=\omega / \Omega$. A careful analysis shows that the spirals are of constant angle, but they are not logarithmic spirals. We define the spiral angle $\psi$ as the angle made when the locus of centers crosses a circle. This angle may be calculated from the slope $\mathrm{d} f / \mathrm{d} g$ where $f(\eta, \beta)$ and $g(\eta, \beta)$ are the solutions given in Eqs. (2.4) and (2.5) of [2] where $\alpha=\sqrt{R / 2}$ is replaced by $\beta=\sqrt{(1+2 \sigma) R / 2}$. Evaluation of this slope gives the pleasingly simple formula for the spiral angles

$$
\psi(R, \sigma)=\frac{\sinh \beta+\sin \beta}{\sinh \beta-\sin \beta} .
$$

A sample plot of the Reynolds number variation of the spiral angles at the selected values $\sigma=$ $\{-0.4,-0.35,-0.2,0.2\}$ is given in Fig. 1. Also included is the limiting $45^{\circ}$ angle achieved as $\sigma \rightarrow \infty$.

The online version of the original article can be found under doi:10.1007/s00707-014-1239-5.

P. D. Weidman $(\varangle)$

Department of Mechanical Engineering, University of Colorado, Boulder, CO 80309-0427, USA

E-mail:weidman@colorado.edu 


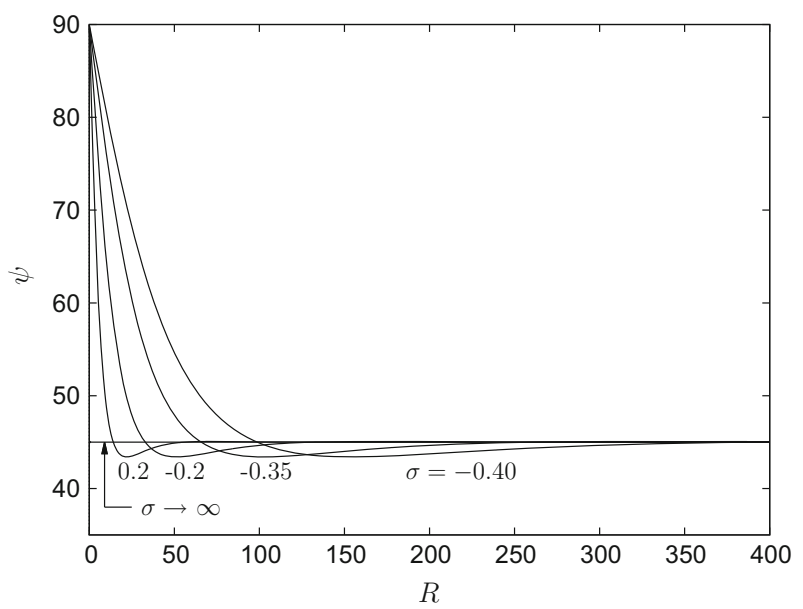

Fig. 1 Reynolds number variation of the angle $\psi$ formed by the locus of centers calculated at the indicated values of $\sigma$. The limiting angle as $\sigma \rightarrow \infty$ is $45^{\circ}$

\section{References}

1. Berker, R.: Intégration des équations du mouvement d'un fluide visqueux incompressible. In: Flügge, S. (ed.) Encyclopedia of Physics, vol. VIII/2, pp. 1-384. Springer, Berlin (1963)

2. Weidman, P.D.: Offset rotating plates in a uniformly rotating fluid. Acta Mech. 226, 1123-1131 (2015) 